\title{
TRANSFER IM FREMDSPRACHENUNTERRICHT AUS DIDAKTISCHER SICHT
}

\begin{abstract}
Vorbemerkung
Der Transfer gilt als eine sehr wichtige Erscheinung im Lernprozess. In den letzten Jahrzehnten wurde diese grundsätzlich sprachbezogen reflektierte Erscheinung in der Fremdsprachendidaktik angesichts der gegenwärtigen theoretischen Grundlagen sowie der Erziehung zur Mehrsprachigkeit in zusätzlichen lernerbezogenen Dimensionen aufgearbeitet. Im vorliegenden Artikel wird nach einem kurzen Rückblick auf das gegenwärtige Verständnis des Transfers in der Fremdsprachendidaktik eingegangen. Vor dem Hintergrund einiger psycholinguistischer Erkenntnisse und lerntheoretischer Überlegungen wird der Prozess des Transfers grundsätzlich in der didaktischen Perspektive diskutiert. Dabei werden punktuelle Erscheinungen des Transfers sowohl in der Relation zwischen der Muttersprache und Fremdsprache als auch in der Perspektive der Erziehung zur Mehrsprachigkeit in Polen hauptsächlich im Kontext Deutsch nach Englisch erwogen.
\end{abstract}

\section{Zur Auslegung des Transfers}

„Transfer bedeutet die Aktualisierung und Anwendung bereits erworbener Kenntnisse und Fertigkeiten auf neue Handlungsstrukturen, die analogen Charakter tragen. Dank der Wirksamkeit des Transfers wird ein ständiges und vollständiges Neulernen unnötig. Dem Lerner bleibt erspart, in bestimmten Situationen immer wieder von neuem adäquate Handlungsreihen aufzubauen“ (Krabe, Piepho 2000: 128). Diese Definition weist auf die wichtige Stellung des Transfers und seine lernerleichternde Rolle beim Fremdsprachenlernen hin.

Der Transfer wird grundsätzlich in zwei Varianten gedeutet. Er kann einerseits unbewusst verlaufen. Andererseits wird er auch als ein Prozess aufgefasst, den man durch bestimmte Vorgehensweise fördern kann. Dem unbewussten und dem be- 
wussten Transfer liegt der gleiche Mechanismus zugrunde. Das Ergebnis der Übertragung kann aber beim unbewusst vollzogenen Transfer entweder positiv oder negativ sein Šumilova (1983: 113). Beim negativen intralingualen also innerhalb der gelernten Fremdsprache zustande kommenden Transfer spricht man von retroaktiver Hemmung, wenn das bereits Gelernte durch neu Gelerntes beeinträchtigt wird und von proaktiver Hemmung, wenn das bereits vorhandene Wissen das neu zu Erwerbende beeinflusst (vgl. Nęcka/Orzechowski/Szymura 2006:635). Bei dem bewusst geförderten Transfer, der durch die didaktischen Maßnahmen erzielt werden soll, wird immer das positive Ergebnis der Übertragung angestrebt. Im Folgenden wird das Augenmerk grundsätzlich auf den interlingualen also auf den zwischensprachlichen Transfer gerichtet.

\section{Von der produktorientierten zur prozessorientierten Deutung des Transfers}

Die Anfänge der Auseinandersetzung mit der Transfererscheinung in der Fremdsprachendidaktik sind bereits mit der Konzipierung der audiolingualen Methode zu verzeichnen. Da das lerntheoretische Paradigma der audiolingualen Methode auf der behaviouristischen Lerntheorie basierte, wurde das Konzept der Sprachvermittlung auf die Ausbildung von Reiz-Reaktion-Verbindungen weitgehend reduziert. Vor diesem Hintergrund wurde auch die Transfererscheinung gedeutet, wobei die partielle Identität der neuen Situation mit der alten als die Basis der Übertragung aufgefasst wurde. Ihre Auswirkung wurde von Lado (1957: 59), wie bekannt, in Form der sog. Kontrastivhypothese formuliert. Diese Hypothese besagte, dass der Transfer von Strukturen aus der Muttersprache in die Fremdsprache einerseits das Erlernen der fremdsprachlichen Strukturen erleichtern, andererseits aber auch erschweren kann. Man nahm an, dass diejenigen Strukturen, die gleich sind, aus der Muttersprache übertragen werden und als Lernerleichterung wirken. Lernprobleme entstehen bei Strukturen, die in den Sprachen unterschiedlich sind. Nach dieser Hypothese, so behauptete man, ist es möglich aufgrund von kontrastiven Untersuchungen Strukturen zu bestimmen, die den Lernern Schwierigkeiten bereiten werden. Diese Strukturen sollten also durch kontrastive Studien festgelegt und in den Lehrmaterialien weitgehend berücksichtigt werden. Ohne weiter auf dieses didaktische Konzept einzugehen, ist festzuhalten, dass sich diese didaktische Auslegung des Transfers grundsätzlich auf die negativen Folgen der Übertragung aus der Muttersprache im Bereich der Strukturmuster konzentrierte, also auf die Interferenz. Die prädiktive Kraft der kontrastiven Hypothese wurde bereits in den 70er Jahren in Frage gestellt. Die vereinzelten Belege, die auf die Enge der auf das Gefüge der äußeren Faktoren reduzierten, behavioristischen Deutung des Transfers hinwiesen, wurden durch die laufenden Untersuchungen im Bereich des Zweitsprachenerwerbs 
untermauert. Ein Überblick über die Änderungen bei der Deutung des Transfers ist u.a. in dem Artikel von Sadownik (1994) zu finden.

Die Fremdsprachendidaktik der 70er Jahre erkannte als wichtig, die Übertragung innerhalb des Unterrichtsprozesses zu fördern, so dass man die während der Unterrichtsstunde die nach der Übungsphase folgende Phase als Transferphase bezeichnete. Diese auf simulierte Kommunikation ausgerichtete Phase sollte den Transfer des eingeübten Sprachmaterials in der außerunterrichtlichen Wirklichkeit begünstigen. Man erkannte, dass das eingeübte Sprachmaterial von selbst nicht übertragungsfähig wird. Auf diese Notwendigkeit macht auch Meißner (2001: 1) mit Verweis auf die Generalisationstheorie aufmerksam, indem er behauptet, dass ,der positive Transfer sich nicht von alleine einstellt, sondern der gezielten Übung bedarf".

Die Tendenz den Transfer breiter aufzufassen, wurde auch durch die lerntheoretischen Auslegungen untermauert. Die gegenwärtige Interpretation der Lernprozesse in der Fremdsprachendidaktik ist dem Lernparadigma der kognitiven Lerntheorie stark verpflichtet. Nach dieser Theorie wird das Lernen als ein Prozess gedeutet, während dessen die aufgenommenen Informationen in die bereits vorhandenen integriert werden.

Im Umfeld der kognitivistischen Auslegung der Lernprozesse wird also das Augenmerk auf die Rolle der bereits vorhandenen Wissensbestände im Verarbeitungsprozess gerichtet, denn der Lernprozess bedeutet eine Modifizierung bzw. Erweiterung vorhandener Wissensstrukturen. Bei dieser Deutung sind die Bezüge bereits bei der Erlernung der ersten Fremdsprache vielfältig zu deuten, da die Wissensstrukturen sowohl das deklarative als auch das prozedurale Wissen umfassen. Bei der Erlernung weiterer Sprachen ist die Übertragung noch wesentlich komplexer zu deuten.

\section{Sprach- und kulturbezogener Transfer}

Apeltauer (1987: 30) behauptet, dass ,,beim Erwerb einer zweiten Sprache der Muttersprache eine Schlüsselfunktion zu [kommt], da einerseits auf die Fähigkeiten, die beim Erstsprachenerwerb entwickelt wurden, zurückgegriffen werden muss, andererseits die Entfernung bzw. Nähe zur Zielsprache Auswirkungen auf den Lernprozess hat".

Die negativen Folgen des sprachbezogenen Transfers sind am deutlichsten an den geschlossenen Subsystemen der zielsprachigen Lernerproduktionen zu erkennen. Auf die negative Auswirkung dieser Übertragung also auf Interferenzfehler z.B. im Bereich des Wortakzents, die auf die Muttersprache zurückgeführt werden können und die es dem Beobachter der Fremdsprachenlerner ermöglichen, die Muttersprache dieser Schüler zu erkennen ,,selbst wenn kein Wort in ihr gesprochen würde" (vorausgesetzt, die Sprache wäre dem Beobachter nicht unbekannt) (Wilkins (1976: 244) verwies man auch in den Anfängen der wissenschaftlich fundierten 
Fremdsprachendidaktik also in der bereits erwähnten audiolingualen Methode. Es werden auditive, artikulatorische und intonatorische Muster der Muttersprache auf die Fremdsprache übertragen. Die überwiegende Zahl der phonetischen Fehler ist das Ergebnis der Interferenz einschließlich der Schriftinterferenz (Dieling 1991: 111; Darski 2010: 44f.) ${ }^{1}$. Bei der zweiten gelernten Fremdsprache kommt auch eine zusätzliche Übertragung in diesem Bereich zustande. Es werden bestimmte Artikulationsmuster aus der ersten gelernten Fremdsprache übertragen. So setzen manche polnischen Grundschulkinder zu Anfang des DAF-Unterrichts, nachdem sie bereits drei Jahre lang Englisch gelernt haben, einen Teil der Artikulationsmuster ein, die auf die englische Sprache zurückgehen. Vereinzelt kommt es auch vor, dass selbst fortgeschrittene Studenten, die Englisch als erste Sprache intensiv gelernt haben und als zweite Sprache Deutsch lernen, z.B. die Aussprache des deutschen „r“ - Lautes durch das englische „r"ersetzen. In diesem Kontext ist bereits auf die von Edmondson (2001: 138) formulierte L1-Transfer-Vermeidungsstrategie hinzuweisen. Dieses Verhaltensprinzip besagt, wenn, wie der Forscher hinzufügt, andere Faktoren außer Acht gelassen werden „Vermeide Transfer aus der Muttersprache zugunsten des Transfers aus anderen Fremdsprachen". Die Vermeidungsstrategie ist insbesondere im Anfängerunterricht zu konstatieren. Bei den beobachteten Grundschulkindern wurde die Übertragung im Bereich der Aussprache im Laufe des Unterrichtsjahres deutlich abgeschwächt und verschwand ungefähr nach einem Unterrichtsjahr. Die Einwirkung der ersten gelernten Fremdsprache auf die zweite (gelernte Fremdsprache) „erreicht aber meistens nicht die Einwirkungsstärke der Muttersprache, selbst wenn die erste Fremdsprache strukturell ähnlicher als die Muttersprache ist" (Reinfried 1998: 32).

In der Fachliteratur wurden zahlreiche Beispiele zusammengestellt, die die Übertragung aus der Muttersprache in die Fremdsprache mit negativem Ergebnis im grammatischen und lexikalischen Bereich belegen. Hierbei ist z.B. auf eine eingehende Deutung der Interferenzfehler von Germanistkstudenten, die von Lietz (1996: $76 \mathrm{ff}$.) vorgenommen wurde, zu verweisen. Beispiele für den negativen Transfer im grammatischen Bereich beim Erlernen des Deutschen nach Englisch durch polnische Lerner z.B.wurden von Kubiczek (2006) zusammengestellt. Auf zahlreiche Möglichkeiten der Bewusstmachung und Förderung der Transfers bezogen auf die sprachlichen Subsysteme im DaF-Unterricht nach Englisch für polnische Lerner gehen Wille, Wawrzyniak (2001) ein.

Inwieweit jedoch der Transfer bei der Füllung der Begriffe zustande kommen kann, ist offensichtlich nur indirekt zu erforschen. Die vorhandenen psycholinguistischen Erkenntnisse zum Konstrukt des mentalen Lexikons geben aber Aufschluss darüber, worauf diese Übertragung zurückzuführen ist. Es besteht gegenwärtig ein weitgehender Konsensus darüber, dass das mentale Lexikon nicht nach der von der

${ }^{1} \mathrm{Zu}$ den einzusetzenden didaktischen Verfahren vgl. z.B. Darski (1993). 
Linguistik angebotenen Beschreibung organisiert ist. Das mentale Lexikon hat die Form eines Netzwerks. Die einzelnen Einträge (die Knoten des Netzwerks) sind miteinander verbunden. Semantisch zusammenhängende Einträge liegen näher beieinander. Diese Nähe wird über die Zahl der gemeinsamen Eigenschaften definiert. In diesem strukturierten Sprachennetzwerk werden ,alle Elemente, Einheiten und Strukturen der neuen Sprachen verankert“ (Neuner 2003:19). Dies bedeutet, dass beim Erlernen einer Fremdsprache neue Repräsentationen für bereits vorhandene Konzepte gelernt werden. Die Sprachen werden also nicht getrennt gespeichert, sondern aufeinander bezogen (vgl. hierzu auch Stork 2003: 76f.). Man betont, dass dabei so wenig wie möglich, neue Repräsentationen und Verbindungen geschaffen werden. Dies wird durch die allgemeinen kognitiven Prinzipien der mentalen Repräsentation sowie den Abbau des kognitiven Verarbeitungsaufwandes bedingt (Ecke, Hall 2000: 30). Der fremdsprachige Lerner versteht die Wortbedeutungen auf der Grundlage eigener soziokulturell geprägter Wissensschemata. Dabei ist anzumerken, dass die Lerner bis in weit fortgeschrittenene Stadien ihrer Zweitsprachenverwendung zur Bedeutungsfindung ihre muttersprachigen Konzepte aktivieren „sofern sie nicht durch ausschließende Kontexte, negatives Feedback des Kommunikationspartners, deutlich wahrnehmbare kulturelle Kontraste usw. daran gehindert werden. Erst wenn solche oder ähnliche Bedingungen vorliegen, wird der Lerner gezwungen L2 oder L2 ähnliche Konzepte in seine Lernersprache zu integrieren“ (Börner, Klaus 1994: 9). Bezogen auf das Erlernen einer zweiten Fremdsprache entsteht in diesem Zusammenhang die Frage, inwieweit sich die Bedeutungsfindung bei der weiteren gelernten Fremdsprache ändert? Unter welchen Bedingungen wird das integrierte L2 Konzept dem L1 Konzept vorangestellt? In diesem Bereich gibt es meines Wissens noch keine Erkenntnisse.

Ein immer noch zu untersuchendes Gebiet in Bezug auf die Transferprozesse stellt der pragmatische Bereich dar. Die deutsche Sprachhandlung Kannst du mir sagen, wie spät es ist? wird von einem Deutsch lernenden Polen ohne weiteres mit Bezug auf das vorhandene Alltagswissen verstanden. Das Sprachhandlungswissen im Bereich der Muttersprache kann also das Verstehen der Illokution in der Fremdsprache positiv beeinflussen. Die Unterrichtserfahrung lehrt aber, dass sich der Transfer der Fähigkeiten nicht immer vollzieht. So ist auch z.B. nicht immer in diesem Bereich mit positivem Transfer bei der schriftlichen Produktion von Texten zu rechnen. Obwohl z.B. die fremdsprachlich fortgeschrittenen Studenten bereits während ihrer muttersprachlichen Ausbildung oft mit den Textsorten Erörterung oder Kommentar zu tun hatten, stellt sich der Transfer in Bezug auf die Struktur dieser Textsorten nicht automatisch ein. Wahrscheinlich wird die Übertragung durch das Ausrichten der Aufmerksamkeit auf die grammatische und lexikalische Realisierung der Aussagen so weit gehemmt, dass der Transfer nicht zustande kommt bzw. ist die Sprachbewusstheit in diesem Bereich nicht dermaßen entwickelt worden ist, dass die bewusste Übertragung hinsichtlich der Textstruktur erfolgen kann. 
Die Gründe dafür sind, so kann angenommen werden, darin zu suchen, dass der fremdsprachliche Unterrichtsprozess immer noch recht isoliert verläuft. Dabei werden die bereits vorhandenen metasprachlichen Vorkenntnisse aus dem Muttersprachenunterricht, bzw. aus der bereits gelernten Fremdsprache kaum aufgegriffen. Die bewusste Auseinandersetzung mit den Sprachhandlungssequenzen könnte einerseits den positiven Transfer begünstigen und damit auch die Entwicklung der Sprachbewusstheit fördern, andererseits für die vorhandenen Unterschiede im Bereich der Sprachhandlungsstrukturen der jeweiligen Textsorten in den verschiedenen Sprachen sensibilisieren. Der Weg, der zur Förderung des Transfers führen kann, ist also in der Entwicklung der Sprachbewusstheit zu sehen, die auf dem bereits vorhandenen Wissen im Bereich der Muttersprache aufbaut und in dem oben erwähten Beispiel auf die Abfolge der Sprachhandlungen in einer Textsorte fokussiert ${ }^{2}$. Anzumerken ist dabei, dass die in Polen gültigen Rahmenrichtlinien im Bereich des Polnischunterrichts die Förderung von Sprachbewusstheit weitgehend einbeziehen und, wie man annehmen kann, auch durch die Umsetzung im Unterricht günstige Grundlagen für die Intergration, also auch für die Förderung des Transfers im Fremdsprachenunterricht schaffen können ${ }^{3}$. Anzunehmen ist auch, dass die kognitive Verarbeitung dem Lerner seine Lernhypothesen und die vollzogene Übertragung bewusst machen und zugleich fördern kann. In dieser Erkenntnisperspektive deutet man aber auch inzwischen fehlerhafte Strukturen, die in Folge des Transfers gebildet werden. Meißner (2001: 3) weist darauf hin, dass sie auch zum Lernfortschritt beitragen können, da sie als falsche und zugleich zu modifizierende Hypothesen aufrecht erhalten werden. Sie sollten aufgegriffen werden und den Ausgangspunkt für die Entwicklung der reflektiven Haltung bilden. Hierbei sind vor allem die bereits in der Fremdsprachendidaktik betonten konstruktiven Potentiale des Sprachvergleichs zu nutzen, die durch entsprechend formulierte Aufgaben gestützt werden können. Es ist zu erwähnen, dass die Übertragung sich nicht nur auf das verbale, sondern auch auf das nonverbale Verhalten beziehen kann. In diesen beiden Bereichen kann jedoch die direkte Übertragung von Sprachhandlungen aus der Muttersprache in die Zielsprache zu unangemessenen Interaktionen, also zu interkulturellen Interferenzen führen. Allerdings sollte dabei nicht vergessen werden, dass die kontrastive Vermittlung des Wissens „über Sprechintentionen und ihre Realisierungen im Unterricht“ nur als Ausgangspunkt gelten kann. Dieses Wissen ,muss immer lückenhaft bleiben und kann höchstens als beispielhaft dargestellt werden. Als Ziel gilt eher ein konstruktiver Umgang mit interkulturellen Situationen (Erschliessungskompetenz)“. (Jandok, Muller-Jacquier 2008: 156). Die reflexive Haltung die durch den Vergleich

\footnotetext{
${ }^{2}$ Die Grundlagen für die Verfahren in diesem Bereich sowie im strukturbezogenen Bereich innerhalb der Textsorten sind in der kontrastiven deutsch-polnischen Grammatik bei (Engel 2000: 120ff.) zu finden.

${ }^{3}$ Vgl. die gültigen Richtlinien für den Schulunterricht: Podstawa Programowa dla gimnazjów i szkół ponadgimnazjalnych, których ukończenie umożliwia uzyskanie świadectwa dojrzałości po zdaniu egzaminu maturalnego. Dziennik Ustaw poz. $17 \mathrm{nr} 4 \mathrm{z}$ roku 2009.
} 
der eigen- und fremdkulturellen Kommunikationsabläufe zu demselben Thema oder der gleichen dominanten Sprachhandlung anstrebt wird, kann also auch für die Grenzen einer solchen Übertragung sensibilisieren, die sich, wie oben angemerkt, aus den kulturbezogenen Unterschieden ergeben, um der Übertragung mit negativem Ergebnis auch in diesem Bereich entgegenzuwirken. Man kann annehmen, dass das Erlernen der zweiten Fremdsprache stärker zum Vergleichen stimuliert und somit auch die Sprachbewusstheit stärker fördern kann.

\section{Sprachlernbezogener Transfer}

Die gegewärtige Aufarbeitung des Tranfers umfasst auch die lernerbezogene Perspektive ${ }^{4}$. Mit Verweis auf Odlin (1989: 139ff.) geht Reinfried (1998: 30) auf diese wichtige Wende ein und schreibt dazu Folgendes „Der Transfer [wurde] (...) als ein Zusammenspiel von objektiven, sprachkontrastiven Faktoren mit subjektiven, lernersprachlichen und lernerstrategischen Faktoren gesehen. Er wird heute überwiegend als Lösungsweg betrachtet, der mit der Vermeidung fremdsprachiger Strukturen konkurriert und von bestimmten subjektiven Voraussetzungen der Lernenden abhängt. Dazu gehören folgende Persönlichkeitsmerkmale: Risikobereitschaft erhöht die Neigung zum Transfer, während Ängstlichkeit sie reduziert; eine ausgeprägte Empathie verringert tendenziell die muttersprachlichen Einwirkungen auf die fremdsprachliche Aussprache“.

In diesem Sinne kommt also beim Umgang mit der Transfererscheinung im Fremdsprachenunterricht der Förderung von Metakognition eine wichtige Stellung zu.

Dabei wird insbesondere an die Reflexion des Lerners in Bezug auf das vorhandene Personen-, Aufgaben- und Strategiewissen sowie dessen Erweiterung gedacht. Das bewusst gemachte subjektive Wissen sollte um die dem Kenntnisstand angepassten und didaktisch aufgearbeiteten lerntheoretischen Erkenntnisse bereichert werden. (Chan 2000: 74). Es ist also notwendig, das metakognitive Wissen des Fremdsprachenlerners in Bezug auf die kontinuierliche Überwachung und Bewertung der Lernprozesse zu fördern. Dies bedeutet, dass das bereits vorhandene und unbewusst benutzte prozedurale Wissen bewusst gemacht und zugleich modifiziert bzw. ergänzt werden sollte. Im Folgenden wird kurz auf den Bereich des Strategiewissens eingegangen. Die Förderung dieses Wissens, das sich u.a. auf die Entwicklung der metakognitiven Kontrollprozesse zur Lernsteuerung bezieht, erfordert die Nutzung der Unterrichtssituationen, in denen der Lerner zur Reflexion über die von ihm gewählte Lernstrategie bei der Bearbeitung der Lernaufgabe durch den Lehrer angeleitet wird.

\footnotetext{
${ }^{4}$ Die verschiedenen Dimensionen der Transfererscheinung im Fremdsprachenunterricht wurden von Edmondson (2001) zusammengestellt.
} 
Im allgemeinen wird angenommen, dass als Strategien sowohl routinisierte Handlungssequenzen gelten können, als auch Handlungen denen bewusste Entscheidungen zugrunde liegen. In der didaktischen Auslegung, die dem kognitiven Verarbeitungsschema verpflichtet ist, sind die Lernstrategien als zielführende Handlungen aufzufassen, die zunächst bewusst eingesetzt und dadurch Einsatz allmählich automatisiert werden können. Sie bleiben jedoch bewusstseins- und dadurch auch zugleich evaluationsfähig. Da sie als Teil des prozeduralen Wissens aufzufassen sind, ist es möglich, dass sie nach der Phase der Routinisierung flexibel genutzt und modifizierungsfähig werden (vgl. hierzu auch Baumert 1993: 329).

Die Langfristigkeit und Explizitheit schulischen Lernens, die auf systematische und langfristige Lernprozesse kumulativer Art angelegt ist, begünstigt nach Baumert (1993: 331) „die Entwicklung der Aufmerksamkeit für eigenes Lernen und damit auch den bewußten Strategieerwerb (...) Das Strategiewissen ist anfänglich intern wenig strukturiert; im Laufe schulischen Lernens und mit der Verbreitung der verfügbaren Wissensbestände sollte sich jedoch das Strategierepertoire ausdifferenzieren, so daß der Einsatz von Lernstragien ein zunehmend flexibler Anpassungsprozess werden kann“. Wenn dies nicht zustande kommt, ist die Übertragung in einem recht bescheidenen impliziten Ausmaß möglich.

Die Grenzen der Übertragung bei der sprachlichen Verarbeitung werden offensichtlich durch die strukturellen Eigenschaften der gelernten Sprachen determiniert. Meißener (2001: 10) schreibt hierzu wie folgt: „Obwohl es sich hier um einen Transfer von Lernerfahrungen handelt, ist dieser, (...) keineswegs sprachenneutral. Vielmehr ist davon auszugehen, daß jede Sprache einen Lerngegenstand eigener Art ausbildet. $\mathrm{Zu}$ nennen ist zunächst die jeweilige sprachtypologische Eigenart der Zielsprache, die einen eigenen methodischen Zugriff verlangt". Mit Verweis auf die Untersuchungsergebnisse von Lutjeharms (1988: 254) betont auch Wolff (2002: 266f.), dass „Muttersprachler des Englischen (einer isolierenden Sprache) [...] beim Lesen deutscher Texte besondere Schwierigkeiten [haben], weil sie es versäumen, auch die Wortenden zu fixieren, die im Gegensatz zum Englischen sehr viele grammatische Informationen enthalten. (...) Ebenso müssen L2-Leser des Deutschen lernen, bestimmte Funktionswörter, z.B. den Artikel genauer zu fixieren, da seine verschiedenen Formen meist mehrere grammatische Informationen enthalten. Auch hier müssen Lerner also neue Strategien entwickeln, die durch die strukturellen Gegebenheiten der neuen Sprache bedingt sind." Für die Deutsch lernenden Polen bedeuten die trennbaren Verben bei der Rezeption der deutschsprachigen Sätze eine Erweiterung der bereits vorhandenen Rezeptionsstrategien, da in der polnischen Sprache das Arbeitsgedächtnis durch die Präfixform des Verbs weniger belastet wird. Das gleiche gilt bei der Verarbeitung der Sätze mit der finiten Form des Verbs, da sie im Polnischen durch die Sufixform des Verbs ausgedrückt wird.

Nützlich können aber sicher andere beim Erlernen der ersten Fremdsprache bereits entwickelte Sprachverarbeitungsstrategien im Bereich der Rezeption werden. 
Dabei sind u.a. folgende Strategien zu erwähnen: der Rückgriff auf die verschiedenen Lesestile, die Aktivierung des Vorwissens, die Haltung, den Text weiterzulesen, weiterzuhören (limitierte Aufmerksamkeit), auch wenn nicht alles verstanden wurde. Wer beim Erlernen der Vokabeln Erfolge mit dem Einsatz von Vokabelkartei beim Lernen der ersten Fremdsprache erzielt hat, wird diese Lernstrategie sicher auch bei der zweiten Fremdsprache einsetzen. Der Transfer von Lernerfahrungen kommt grundsätzlich zustande, wenn diese beim Erlernen der ersten Sprache eher positiv waren. Die kurz angelegte Befragung unter den Lernern im Lyzeum, die Deutsch als zweite Fremdsprache nach Englisch lernen, ergibt, dass die subjektiv von den Lernern eingeschätzte und lernerleichternde Übertragung sich grundsätzlich auf das Wortschatzlernen bezieht. Die aufgezählten Beispiele gehen aber selten über die mnemotechnische Nutzung der englischen Vokabeln hinaus. Die Hilfe des Lehrers bei der Förderung der Übertragung steht in diesem Bereich oft noch aus. Man findet auch kaum Lehrwerke, die die Lerner auf diese Möglichkeiten explizit hinweisen, wobei gerade die explizite Arbeit an Lernstrategien in der ersten Fremdsprache als eine wichtige Voraussetzung für eine effiziente Übertragung des prozeduralen Wissens gilt.

\section{Abschließende Bemerkungen}

Es ist unmöglich, im Rahmen eines Artikels die vielfältigen Dimensionen des Transferprozesses im Fremdsprachenunterricht und seine Auswirkungen darzustellen. Daher wurde versucht, lediglich bestimmte Schwerpunkte der didaktischen Arbeit, die sich auf diese Erscheinung bezieht, zu reflektieren. Der bewussten Auseinandersetzung der Lerner mit den Sprachphänomenen und dem Lernprozess, die den didaktisch stimulierten Vergleich als Ausgangspunkt für die Verarbeitung des Wissens nutzt und die Erkenntnis der Grenzen sowie der Möglichkeiten der Übertragung von Wissen fördert, kommt dabei eine wichtige Rolle zu. Die Potenziale des Transfers sollten auch durch die entsprechende Verzahnung im Bereich der curricularen Arbeiten und durch die in den Lehrwerken vorgeschlagenen Wege, die über den subsytematischen Bereich der gelernten Fremdsprachen hinausgehen, gefördert werden und somit den Lehrern helfen, einen integrativen Unterricht zu gestalten.

\section{Literatur}

Apeltauer, E. (1987): Gesteuerter Zweitsprachenerwerb. Voraussetzungen und Konsequenzen für den Unterricht. München: Hueber Verlag.

Baumert, J. (1993): Lernstrategien, motivale Orientierung und Selbstwirksamkeits-überzeugungen. In: Unterrichtswissenschaft 21, S. 327-354. 
Börner K., Vogel W. (1994): Mentales Lexikon und Lernersprache. In: K. Börner, W. Vogel (Hrsg): Kognitive Linguistik und Fremdsprachenerwerb, Tübingen: Narr Verlag, S. 1-17.

Chan W.M. (2000): Metakognition und der DaF-unterricht für asiatische Lerner. Münster u.a.: Waxmann Verlag.

Darski, J. (1993): Jak uczyć fonetyki? In: Polska w zjednoczonej Europie. Nauczanie Języków Obcych. Lublin: Wydawnictwo Uniwersytetu Marii Curie-Skłodowskiej, S. 27-33.

Darski, J. (2010): Jak można perfekcyjnie opanować język obcy. In: M. Pawlak, E. Waniek-Klimczak: Mówienie w języku obcym - sukcesy i porażki uczenia się i nauczania. Poznań-Kalisz-Konin: Wydział Pedagogiczno-Artystyczny UAM w Kaliszu, Uniwersytet im. A. Mickiewicza w Poznaniu, S. 37-50.

Dieling, H. (1991): Nicht bagatelisieren. Phonetische Fehler im FU. Deutsch als Fremdsprache 2, S. 111-115.

Ecke P., Hall CH. J. (2000): Lexikalische Fehler in Deutsch als Drittsprache. Translexikalischer Einfluß auf drei Ebenen der mentalen Repräsentation, In: Deutsch als Fremdsprache 37, S. 30-36.

Edmondson, W.J. (2001): Transfer beim Erlernen einer weiteren Fremdsprache: Die L1-TransferVermeidungsstrategie. In: K. Aguado, C. Riemer: Wege und Ziele. Zur Theorie, Empirie und Praxis des Deutschen als Fremdsprache (und anderer Sprachen). Hohengehren: Schneider Verlag, S. 137-154.

Engel, U. (2000): Deutsch-polnische kontrastive Grammatik. Bd. 1, Warszawa: Wydawnictwo Naukowe PWN.

Jandok, P., Müller-Jacqiuer, B. (2008): Deutsch als Fremdsprache-Lehrwerke und die Vermittlung interkultreller Kompetenzen. Ein wssens- und strategiebezogenes Analyseinstrument. In: Beiträge zur Fremdsprachenvermittlung. Sonderheft 13, S. 151-173.

Krabe U., Piepho H.E. (2000): Fremdsprachenunterricht von A bis Z: praktisches Begriffswörterbuch. Ismaning: Hueber Verlag.

Kubiczek, A. (2006): Grammatik-Transfer im Fremdsprachenunterricht.Vor- und Nachteile. <http://www2. rz.hu-berlin.de/linguistik/institut/syntax/krakau2006/beitraege/kubiczek.pdf> (8.04.2011).

Lado, R. (1957): Linguistics across cultures. Applied linguistics for language teachers. University of Michigan Press.

Lietz G. (1996): Zwischensprachliche Interferenz als Fehlerquelle in schriftlichen Arbeiten polnischer Germanistkstudenten. Glottodidactica XXIV, S. 73-120.

Lutjeharms, M. (1988): Lesen in der Fremdsprache: Versuch einer psycholinguistischen Deutung am Beispiel Deutsch als Fremdprache. Bochum: AKS-Verlag.

Meißner, F.-J. (2001): Transfer aus der Sicht der Mehrsprachigkeitsdidaktik. <http://www.fernunihagen.de/sprachen/kongress/Abstracts/MeissnerDE.pdf> (8.04.2011).

Neuner, G. (1996): Deutsch als zweite Fremdsprache nach Englisch. Überlegungen zur Didaktik und Methodik und zur Lehrmaterialienentwicklung für die „Drittsprache Deutsch“. In: Deutsch als Fremdsprache 4, S. 211-217.

Neuner, G. (2003): Das Konzept der Mehrsprachigkeitsdidaktik. In: B. Hufeisen, G. Neuner (Hrsg.): Mehrsprachigkeitskonzept - Tertiärsprachenlernen - Deutsch nach Englisch. Straßburg: Council of Europe Publishing, S.13-34.

Nęcka, E., Orzechowski, J., Szymura, B. (2006): Psychologia poznawcza. Warszawa: PWN.

Odlin, T. (1989): Language transfer: cross-linguistic influence in language learning. Cambridge: Cambridge University Press.

Podstawa programowa dla gimnazjów i szkół ponadgimnazjalnych, których ukończenie umożliwia uzyskanie świadectwa dojrzałości po zdaniu egzaminu maturalnego (2009): Dziennik Ustaw, nr 4, poz. 17.

Reinfried, M. (1998): Transfer beim Erwerb einer weiteren romanische Fremdsprache. Prinzipielle Relevanz und methodische Integration in den Fremdsprachenunterricht. In: F.-J. Meißner, M. Rein- 
fried, (Hrsg): Mehrsprachigkeitsdidaktik. Konzepte, Analysen, Lehrerfahrungen mit romanischen Sprachen. Tübingen: Günter Narr Verlag, S. 23-44.

Sadownik, B. (1994): Sprachlicher Tranfer im Fremdsprachenunterricht aus der Sicht kognitiver Prozesse. In: Roczniki Humanistyczne 5, S. 77-88.

Stork, A. (2003): Vokabellernen. Tübingen: Narr Verlag.

Šumilova, E.M. (1983): Der Transfer als psychologischer Prozeß. In: Deutsch als Fremdsprache 2, S. 111-115.

Wilkins, D.A. (1976): Linguistik im Sprachunterricht. Heidelberg: Quelle \& Mayer.

Wille, L., Wawrzyniak, Z. (2001): Deutsch nach Englisch im glottodidaktischen Gefüge. Rzeszów: Wydawnictwo Wyższej Szkoły Pedagogicznej.

Wolff, D. (2002): Fremdsprachenlernen als Konstruktion. Grundlagen für eine konstruktivistische Fremdsprachendidaktik. Frankfurt am Main u.a.: Peter Lang Verlag. 